\title{
Variações Foliares em Grupos Funcionais Vegetais de uma Paisagem de Restinga, Pernambuco-Brasil
}

\author{
Maria das Graças Santos das Chagas ${ }^{1^{*}}$ \\ Milena Dutra da Silva ${ }^{{ }^{*}}$ \\ Josiclêda Domiciano Galvíncioº \\ Rejane Magalhães de Mendonça Pimentel ${ }^{3}$
}

Artigo recebido em 27/11/2008 e aceito para publicação em 19/03/2009.

\section{RESUMO}

As paisagens variam em composição e riqueza de espécies vegetais, essas variações são dependentes das condições abióticas ambiente. A permanência das espécies vegetais em uma determinada área depende das estratégias de aclimatação e adaptação que essas espécies possuem. A amplitude da resposta biológica de todas as espécies às condições do ambiente condiciona o tamanho da sua população. Este estudo visou caracterizar e agrupar as características morfológicas foliares das espécies vegetais ocorrentes em uma fisionomia de restinga. Foram detectadas variações morfométricas entre as espécies e entre os indivíduos. Através da análise de agrupamento foram formados três grupos funcionais, onde a principal característica edafoclimática norteadora do agrupamento foi o grau de luminosidade disponível. É provável que as variações fenotípicas observadas nas 15 espécies sejam uma forma de aumentar a captação de luz pela lâmina foliar. Deste modo, as diferenças morfológicas significativas encontradas podem ser consideradas de aclimatação, haja vista estarem contribuindo para a estabilidade funcional destas plantas.

Palavra-chave: Morfologia foliar, Variações fenotípicas foliares, Restinga.

\section{Leaves Variations in Plant Functional Groups of a Landscape of Restinga, Pernambuco-Brazil}

\begin{abstract}
The landscape varies in response to the plant species; these responses are dependent of the abiotic conditions of the environment. The maintenance of these species in a determined area is dependent to the strategies of acclimatation and adaptation that these species posses. The amplitude of the biological response of all these species to the environmental conditions is determinant to the size of the population. This study aimed to characterize and group the leaf morphological characteristics of the plant species which occur in a physiognomy of restinga. Morphological variations were detected among the individuals. The group analysis showed

\footnotetext{
${ }^{1}$ Doutorandas do Programa de Pós-Graduação em Geografia - Universidade Federal de Pernambuco (UFPE). UFPE, Centro de Filosofia e Ciências Humanas, Departamento de Ciências Geográficas. Avenida Professor Moraes Rego, 1235, Cidade Universitária, Recife, Pernambuco. CEP 50670-901. (mgschagas@hotmail.com; dutra_ms@yahoo.com.br).

${ }^{2}$ Universidade Federal de Pernambuco. Departamento de Geografia. (josicleda@pq.cnpq.br).

${ }^{3}$ Universidade Federal Rural de Pernambuco. Departamento de Biologia/Botânica. (pimentel@db.ufrpe.br).

*Autor para correspondência.
} 
the existence of three functional groups, and the main edaphoclimatic characteristic was the light available. It is probable that the phenotypic variations found in 15 species were a way to enhance the light capture by the leaf lamina. In this way, the significant morphological differences can be considered an acclimatation, because it is contributing to the functional stability of these plants.

Key-words: Leaf morphology, Leaf phenotypical variations, Restinga.

\section{INTRODUÇÃO}

As planícies sedimentares, ao longo de sua evolução geomorfológica, adquiriram a forma de cordões arenosos, decorrentes das transgressões e progressões marinhas, apresentando uma topografia complexa e diversificada modelada pela ação dos ventos, das tempestades e das correntes litorâneas (Cantarelli, 2003). Nestas áreas, a vegetação pode variar de campos de gramíneas a florestas, sendo denominadas de restingas, ocupando, aproximadamente, $5.000 \mathrm{~km}$ do litoral brasileiro (Araújo e Lacerda, 1987).

No ecossistema restinga, os fatores que interferem no aspecto geral da vegetação, influentes no desenvolvimento e no crescimento das diversas espécies vegetais, são, principalmente, aqueles relacionados ao solo, como a baixa concentração de nutrientes, baixa capacidade de retenção hídrica e as elevadas concentrações salinas, além de elevadas temperaturas e exposição luminosa (Henriques et al., 1986). Esta configuração ambiental propicia o favorecimento da manifestação da plasticidade fenotípica nas plantas, além da formação de ecótipos (Fuzeto e Lomônaco 2000).

A restinga apresenta uma diversidade fisionômica, mesclando espécies nativas com outras provenientes de Floresta Atlântica, Caatinga e Cerrado (Freire, 1990). Algumas espécies, ao migrarem de outro ambiente para a restinga, foram induzidas a se adaptarem às novas condições ambientais, através de estratégias funcionais, variando suas estruturas (Esteves et al., 2002). Essa habilidade apresentada pelos órgãos vegetativos para alterar caracteres fenotípicos em decorrência da interação com o ambiente contribui para a estabilidade funcional do indivíduo, principalmente quando a plasticidade fenotípica age sobre caracteres ligados à sobrevivência (Reis, 2003).

As espécies presentes nas comunidades vegetais apresentam uma dinâmica que ocasiona uma variação temporal e espacial na composição florística de uma dada área. A variação 
apresentada está diretamente relacionada aos processos ecológicos e evolutivos do ambiente (Crawley, 1997).

Os principais filtros determinantes da permanência e/ou exclusão da vegetação são os fatores abióticos, os quais controlam que espécies, presentes em escala regional, podem representar a vegetação em escala local. Essa representação está intimamente relacionada com a plasticidade fenotípica da espécie em relação ao microclima (Furley e Ratter, 1988). Entre os órgãos vegetativos das plantas, a folha, principal órgão fotossintético, destaca-se por sua elevada capacidade plástica, respondendo pela adaptação dos indivíduos aos mais diversos ambientes, através de modificações químicas, fisiológicas e morfológicas (Monteiro et al., 2005).

A morfoanatomia foliar da flora de ambientes úmidos vem sendo estudada para fornecer subsídios ecológicos, visando caracterizar e apontar alterações nas estruturas funcionais deste órgão (Borger e Wisnienski, 2003; Klich, 2000; Kong, 2001). Entretanto, as espécies remanescentes das restingas brasileiras ainda não estão bem caracterizadas, faltam informações acerca da morfoanatomia dessas espécies, principalmente aquelas relativas à identificação das estruturas funcionais e ambientais que auxiliam na preservação deste ecossistema (Araújo e
Henrique, 1984). Diante do exposto, este trabalho objetivou caracterizar e agrupar as características morfológicas foliares de espécies vegetais ocorrentes em uma fisionomia de restinga no estado de Pernambuco, Brasil.

\section{MATERIAL E MÉTODOS}

Área de estudo - A Reserva Particular do Patrimônio Natural (RPPN) Nossa Senhora do Outeiro de Maracaípe (IBAMA 2000) localiza-se no litoral sul de Pernambuco, município de Ipojuca, sob as coordenadas 0831'48'’s e $35^{\circ} 01^{\prime} 05^{\prime \prime}$ 'W e ocupa uma área de 76,2 ha sobre a planície litorânea. Dessa área, 60,96 ha correspondem à vegetação de restinga e 15,24 ha correspondem à vegetação de mangue, a qual margeia a restinga. A área dista cerca de $5 \mathrm{~km}$ do mar e, de maneira geral, apresenta relevo plano com suaves ondulações. O solo é constituído por Neossolos Quartzarênicos e, apesar de não existirem lagoas perenes na área, alguns trechos do solo podem ficar inundados durante o período chuvoso. O clima, segundo a classificação de Köppen (1948), é do tipo As' tropical chuvoso, com verão seco e menos de $60 \mathrm{~mm}$ de chuva no mês mais seco, com precipitação pluviométrica anual aproximada de 2.000 mm. A estação chuvosa ocorre de abril a setembro, sendo os meses de maio, junho e julho os mais chuvosos; a estação seca vai de outubro a 
março, sendo os meses de outubro, novembro e dezembro os mais secos (INMET, 2005).

\section{Seleção e coleta das espécies - Foram} selecionadas as espécies vegetais mais freqüentes na área, distribuídas seguindo uma faixa no continente, a qual se estende da margem da vegetação de mangue até o interior da RPPN. A localização de todos os indivíduos foi determinada através de GPS. O critério adotado para a classificação dos grupos funcionais, considerando árvores e arbustos, baseou-se em Rizzini (1997). Foram coletadas dez folhas adultas de cada um dos três indivíduos por espécie selecionada, sendo consideradas como folhas adultas àquelas localizadas entre o quarto e o oitavo nó, a partir do ápice do ramo, com lâmina completamente expandida, quando comparadas com as demais folhas do mesmo indivíduo. Todas as folhas encontravam-se expostas ao sol. O material botânico foi acondicionado em sacos plásticos devidamente etiquetados e transportados, em isopor, até o Laboratório de Fitomorfologia Funcional (LAFF) da Universidade Federal Rural de Pernambuco (UFRPE) para processamento.

Processamento dos dados - Foram obtidas imagens digitalizadas das folhas de Chagas, M.G.S.; Silva, M. D.; Galvíncio, J. D. \& Pimentel, R. M. M. todos os indivíduos por espécie, utilizando scanner de mesa. Nestas imagens foram determinados os caracteres foliares utilizados para a caracterização das espécies: forma da borda, margem e ápice foliar, área foliar (AF), comprimento do pecíolo (CP), comprimento do limbo (CL), largura do limbo (LL) índice foliar (IF). O IF (Reis, 2003) foi calculado dividindo-se o valor do comprimento do limbo (CL) por sua largura (LL). O IF caracterizou o formato foliar: folhas com valor de $\mathrm{IF} \cong 1$ (um) são arredondadas, ou seja, isodiamétricas; IF < 1 são mais largas que longas; IF> 1 são, proporcionalmente, mais alongadas. As medidas morfológicas foram realizadas utilizando o programa de análise de imagens Image Tool (Wilcox et al., 2002). A descrição dos tipos morfológicos foliares dos indivíduos seguiu a terminologia de Radford et al. (1974).

Testes estatísticos - A formação de grupos funcionais foi verificada através de testes de agrupamento, utilizando o método de Ward’s, com distância euclidiana.

\section{RESULTADOS}

A forma do limbo foliar das espécies estudadas foi, predominantemente, elíptica, tanto para árvores quanto para arbustos, apresentando, também, a forma oval, oboval e oblonga (Tab. 1). Duas formas de 
ápice foliar foram predominantes entre as espécies estudadas, o acuminado e o agudo, apresentando, ainda, formas contraídas como agudo-arredondado, agudo-acuminado, entre outros (Tab. 1). A base mostrou-se arredondada na maioria das espécies analisadas (Tab. 1).

Tabela 1. Classificação da morfologia foliar em 15 espécies lenhosas mais abundantes em uma área de restinga em Maracaípe, Pernambuco-Brasil.

\begin{tabular}{llll}
\hline Espécie & Forma & Ápice & Base \\
\hline $\begin{array}{l}\text { Combretaceae } \\
\text { Buchenavia capitata } 1\end{array}$ & Oboval & Atenuado & Retuso \\
$\begin{array}{l}\text { Buchenavia capitata } 2 \\
\text { Buchenavia capitata } 3\end{array}$ & Oboval & Arredondado-Retuso & Atenuada \\
& Oboval & Arredondado-Retuso & Atenuada \\
Sapotaceae & & & \\
Manilkara salzmannii 1 & Elíptica-Oboval & Retuso & \\
Manilkara salzmannii 2 & Oboval & Retuso & Atenuada \\
Manilkara salzmannii 3 & Oboval & Retuso & Atenuada \\
& & & Atenuada
\end{tabular}

\section{Fabaceae}

Andira fraxinifolia 1

Andira fraxinifolia 2

Andira fraxinifolia 3

\section{Mimosaceae}

Inga flagelliformis 1

Inga flagelliformis 2

Inga flagelliformis 3

Abarema filamentosa 1

Abarema filamentosa 2

Abarema filamentosa 3

$\begin{array}{ll}\text { Elíptica-Oval } & \text { Agudo } \\ \text { Elíptica-Oval } & \text { Agudo } \\ \text { Elíptica-Oval } & \text { Agudo }\end{array}$

Arredondado

Arredondado

Arredondado

\begin{tabular}{|c|c|}
\hline Elíptica & Atenuado \\
\hline Elíptica & Atenuado \\
\hline Elíptica & Atenuado \\
\hline Elíptica & Agudo \\
\hline Elíptica & Agudo \\
\hline Oblonga & Truncado-Arredondado \\
\hline
\end{tabular}

\section{Myrtaceae}

Myrcia guianensis 1

Myrcia guianensis 2

Oval

Oval

Oval

Myrcia bergiana 1

Oboval

Oboval

Oboval

Myrcia bergiana 3

\section{Humiriaceae}

Saccoglotis mattogrossensis 1

Saccoglotis mattogrossensis 2
Elíptica

Elíptica
Agudo-Arredondado

Agudo-Arredondado

Agudo-Arredondado

Acuminado

Acuminado

Acuminado

Acuminado

Agudo

Acuminado
Arredondado

Arredondado

Arredondado

Arredondado

Arredondado

Arredondado 


\section{Flacourtiaceae}

Casearia javitensis 1

Elíptica

Elíptica

Elíptica

Casearia javitensis 3

\section{Apocynaceae}

Hancornia speciosa 1

Hancornia speciosa 2

Hancornia speciosa 3

$\begin{array}{ll}\text { Oblonga-Elíptica } & \text { Acuminado } \\ \text { Oblonga-Elíptica } & \text { Acuminado } \\ \text { Oblonga-Elíptica } & \text { Acuminado }\end{array}$

Acuminado

Agudo

Agudo

Arredondado

Arredondado

Arredondado

\section{Caesalpinaceae}

Chamaecrista ensiformes 1

Chamaecrista ensiformes 2

Elíptica

Elíptica

Chamaecrista ensiformes 3

Acuminado

Agudo

Agudo
Arredondado

Arredondado

Arredondado
Atenuada

Arredondado

Agudo
Lauraceae

Ocotea gardneri 1

Ocotea gardneri 2

Ocotea gardneri 3

\section{Sapindaceae}

Cupania racemosa 1

Cupania racemosa 2

Cupania racemosa 3

\section{Elaeocarpaceae}

Sloanea guianensis 1

Sloanea guianensis 2

Sloanea guianensis 3

\section{Myrcinaceae}

Rapanea gardneriana 1

Rapanea gardneriana 2

Rapanea gardneriana 3

\author{
Elíptica-Olonga \\ Elíptica-Olonga \\ Elíptica-Olonga \\ Aguda-Acuminada \\ Aguda-Acuminada \\ Aguda-Acuminada
}

Elíptica
Elíptica
Elíptica

Oboval

Oblonga

Elíptica
Aguda-Acuminada

Aguda-Acuminada

Aguda-Acuminada
Arredondada

Arredondada-Aguda

Agudo

Atenuada-Cuneada Atenuada-Cuneada

Atenuada-Cuneada

Atenuada-Cuneada Atenuada-Cuneada Atenuada-Cuneada

Arredondado

Arredondado

Cuneada-Aguda

Os maiores valores para comprimento médio do pecíolo foram determinados em indivíduos da espécie Sloanea cf. guianensis, enquanto que o menor comprimento médio de pecíolo foi encontrado nos indivíduos da espécie

\begin{tabular}{lll} 
Elíptica & Arredondado-Agudo & Atenuada-Cuneada \\
Elíptica & Arredondado-Agudo & Atenuada-Cuneada \\
Elíptica & Arredondado-Agudo & Atenuada-Cuneada \\
\hline
\end{tabular}

Andira fraxinifolia (Tab. 2). Foram encontradas grandes variações nos valores da área foliar entre os indivíduos estudados. A maior área foliar foi observada em Sloanea cf. guianensis e a menor em Hancornia speciosa (Tab. 2). 
O Índice Foliar (IF) indica a relação entre largura e comprimento foliar, informando acerca do investimento do indivíduo em crescimento ou alargamento da lâmina foliar. Todas as espécies apresentaram valores maiores que um, indicando que essas espécies possuem folhas mais longas do que largas, ou seja, elas investem mais no crescimento longitudinal das folhas do que na largura (Tab. 2).

Tabela 2. Morfometria foliar de 15 espécies lenhosas mais abundantes em uma área de restinga, Pernambuco-Brasil. C-Peciolo = comprimento do pecíolo, A-Foliar = área foliar, C-Foliar = comprimento foliar, L-Foliar = largura foliar e IF = índice foliar.

\begin{tabular}{lcrrcc}
\hline Espécies & C-Pecíolo & \multicolumn{1}{c}{ A-Foliar } & \multicolumn{1}{c}{ C-Foliar } & L-Foliar & IF \\
\hline Combretaceae & & & & & \\
Buchenavia capitata 1 & $0,68 \pm 0,27$ & $8,26 \pm 0,85$ & $4,50 \pm 0,33$ & $2,63 \pm 0,09$ & $1,71 \pm 0,12$ \\
Buchenavia capitata 2 & $0,87 \pm 0,15$ & $17,15 \pm 4,60$ & $6,76 \pm 1,27$ & $3,54 \pm 0,46$ & $1,90 \pm 0,22$ \\
Buchenavia capitata 3 & $0,77 \pm 0,13$ & $15,02 \pm 6,71$ & $6,18 \pm 1,58$ & $3,21 \pm 0,82$ & $1,93 \pm 0,18$
\end{tabular}

\section{Sapotaceae}

Manilkara salzmannii 1

Manilkara salzmannii 2

Manilkara salzmannii 3

\section{Fabaceae}

Andira fraxinifolia 1

Andira fraxinifolia 2

Andira fraxinifolia 3

$\begin{array}{ll}0,22 \pm 0,03 & 17,36 \pm 2,02 \\ 0,28 \pm 0,02 & 21,53 \pm 4,58 \\ 0,26 \pm 0,03 & 16,80 \pm 3,04\end{array}$

$1,30 \pm 0,15$

$26,19 \pm 4,34$

$1,50 \pm 0,26$

$25,39 \pm 4,78$

$0,94 \pm 0,23$

$19,33 \pm 2,32$

$8,67 \pm 0,80$

$4,08 \pm 0,23 \quad 2,12 \pm 0,12$

$8,73 \pm 1,03$

$7,66 \pm 0,76$

$3,60 \pm 0,39 \quad 2,42 \pm 0,09$

$3,49 \pm 0,19 \quad 2,20 \pm 0,24$

\section{Mimosaceae}

Inga flagelliformis 1

$0,93 \pm 0,04$

$53,31 \pm 15,01$

$13,58 \pm 2,09$

$5,94 \pm 0,89 \quad 2,30 \pm 0,25$

Inga flagelliformis 2

$0,75 \pm 0,16$

$41,17 \pm 10,28$

$12,30 \pm 1,58$

$0,76 \pm 0,20$

$36,31 \pm 8,99$

$11,39 \pm 1,54$

$4,99 \pm 0,64 \quad 2,47 \pm 0,16$

Inga flagelliformis 3

$0,17 \pm 0,06$

$34,11 \pm 6,33$

$9,04 \pm 0,87$

$4,72 \pm 0,60 \quad 2,42 \pm 0,18$

Abarema filamentosa 1

$0,10 \pm 0,03$

$11,83 \pm 0,70$

$5,52 \pm 0,27$

$5,16 \pm 0,48 \quad 1,76 \pm 0,46$

Abarema filamentosa 2
Abarema filamentosa 3

$0,13 \pm 0,03$

$24,20 \pm 4,63$

$7,34 \pm 0,86$

$3,01 \pm 0,06 \quad 1,83 \pm 0,06$

$4,18 \pm 0,41 \quad 1,75 \pm 0,04$

\section{Myrtaceae}

Myrcia guianensis 1

Myrcia guianensis 2

$0,29 \pm 0,05$
$0,35 \pm 0,10$
$0,34 \pm 0,10$

$16,56 \pm 2,62$

$5,14 \pm 0,54$

$4,09 \pm 0,20 \quad 1,26 \pm 0,11$

Myrcia guianensis 3

$9,46 \pm 1,32$

$4,09 \pm 0,46$

$2,99 \pm 0,15 \quad 1,37 \pm 0,10$

$8,77 \pm 1,67$

$4,33 \pm 0,40$

$2,75 \pm 0,27 \quad 1,58 \pm 0,10$

Myrcia bergiana 1

$0,85 \pm 0,10$

$48,91 \pm 11,38$

$14,66 \pm 1,95$

$4,73 \pm 0,50 \quad 3,10 \pm 0,23$

Myrcia bergiana 2

$0,93 \pm 0,08$

$30,15 \pm 4,27$

$9,99 \pm 1,09$

$4,37 \pm 0,35 \quad 2,28 \pm 0,10$

Myrcia bergiana 3

$0,78 \pm 0,17$

$38,35 \pm 12,48$

$12,41 \pm 2,33$

$4,75 \pm 0,78 \quad 2,61 \pm 0,10$ 


\section{Humiriaceae}

Saccoglotis mattogrossensis 1 Saccoglotis mattogrossensis 2 Saccoglotis mattogrossensis 3

$0,67 \pm 0,16$

$0,63 \pm 0,04$

$1,13 \pm 0,26$

\section{Flacourtiaceae}

Casearia javitensis 1

Casearia javitensis 2

Casearia javitensis 3

\section{Apocynaceae}

Hancornia speciosa 1

Hancornia speciosa 2

Hancornia speciosa 3

\section{Caesalpinaceae}

Chamaecrista ensiformes 1

Chamaecrista ensiformes 2

Chamaecrista ensiformes 3

Lauraceae

Ocotea gardneri 1

Ocotea gardneri 2

Ocotea gardneri 3

Sapindaceae

Cupania racemosa 1

Cupania racemosa 2

Cupania racemosa 3

\section{Elaeocarpaceae}

Sloanea guianensis 1

Sloanea guianensis 2

Sloanea guianensis 3

$$
\begin{array}{r}
0,66 \pm 0,12 \\
0,84 \pm 0,22 \\
0,54 \pm 0,08
\end{array}
$$

$30,56 \pm 8,31$

$41,70 \pm 13,64$

$26,95 \pm 12,42$
$39,32 \pm 10,09$

$43,00 \pm 9,90$

$58,63 \pm 11,74$
$11,63 \pm 1,41$

$11,13 \pm 1,81$

$11,51 \pm 1,51$
$4,70 \pm 0,67 \quad 2,48 \pm 0,18$

$5,23 \pm 0,50 \quad 2,13 \pm 0,30$

$6,87 \pm 0,63 \quad 1,67 \pm 0,15$
$4,15 \pm 0,53 \quad 2,40 \pm 0,14$

$4,43 \pm 0,62 \quad 2,93 \pm 0,28$

$3,76 \pm 0,94 \quad 2,60 \pm 0,38$

$$
0,48 \pm 0,01
$$

$0,41 \pm 0,03$

$49,23 \pm 8,07$

$24,22 \pm 2,95$

$30,64 \pm 2,04$

$15,16 \pm 1,33$

$9,45 \pm 0,92$

$11,66 \pm 0,76$
$4,65 \pm 0,56 \quad 3,29 \pm 0,47$

$3,44 \pm 0,26 \quad 2,75 \pm 0,25$

$3,91 \pm 0,08 \quad 2,98 \pm 0,18$
$1,83 \pm 0,19$
$59,53 \pm 15,40$
$13,19 \pm 1,66$
$6,24 \pm 0,71 \quad 2,13 \pm 0,27$
$1,05 \pm 0,34$
$56,80 \pm 19,84$
$13,11 \pm 2,43$
$6,15 \pm 1,18 \quad 2,15 \pm 0,26$
$1,40 \pm 0,11$
$50,31 \pm 12,33$
$6,04 \pm 0,89 \quad 1,94 \pm 0,15$

$6,88 \pm 0,74 \quad 2,17 \pm 0,16$

$5,57 \pm 1,48 \quad 2,12 \pm 0,43$

$5,60 \pm 0,71 \quad 2,11 \pm 0,16$

\section{Myrcinaceae}

Rapanea gardneriana 1

$0,59 \pm 0,08$

$9,67 \pm 2,36$

$5,37 \pm 0,52$

$2,55 \pm 0,39 \quad 2,13 \pm 0,25$

Rapanea gardneriana 2

$0,67 \pm 0,14$

$17,88 \pm 5,23$

$6,68 \pm 1,08$

$2,55 \pm 0,39 \quad 2,70 \pm 0,70$

Rapanea gardneriana 3

$0,74 \pm 0,07$

$15,27 \pm 4,74$

A análise de agrupamento pelo método de Ward's mostrou a existência de três grupos funcionais (Fig. 1). Analisando o agrupamento de indivíduos, sem considerar a identificação taxonômica das espécies, foi constatado que o grupo 1 está composto por 10 indivíduos, o grupo 2 por 17 indivíduos e o grupo 3 por 18 
indivíduos. Entretanto, ao analisar o composto de quatro espécies e o grupo 2 e agrupamento com a identificação 3 continham sete espécies cada um. taxonômica, observa-se que o grupo 1 está

Tree Diagram for 45 Cases

Ward's method

Euclidean distances

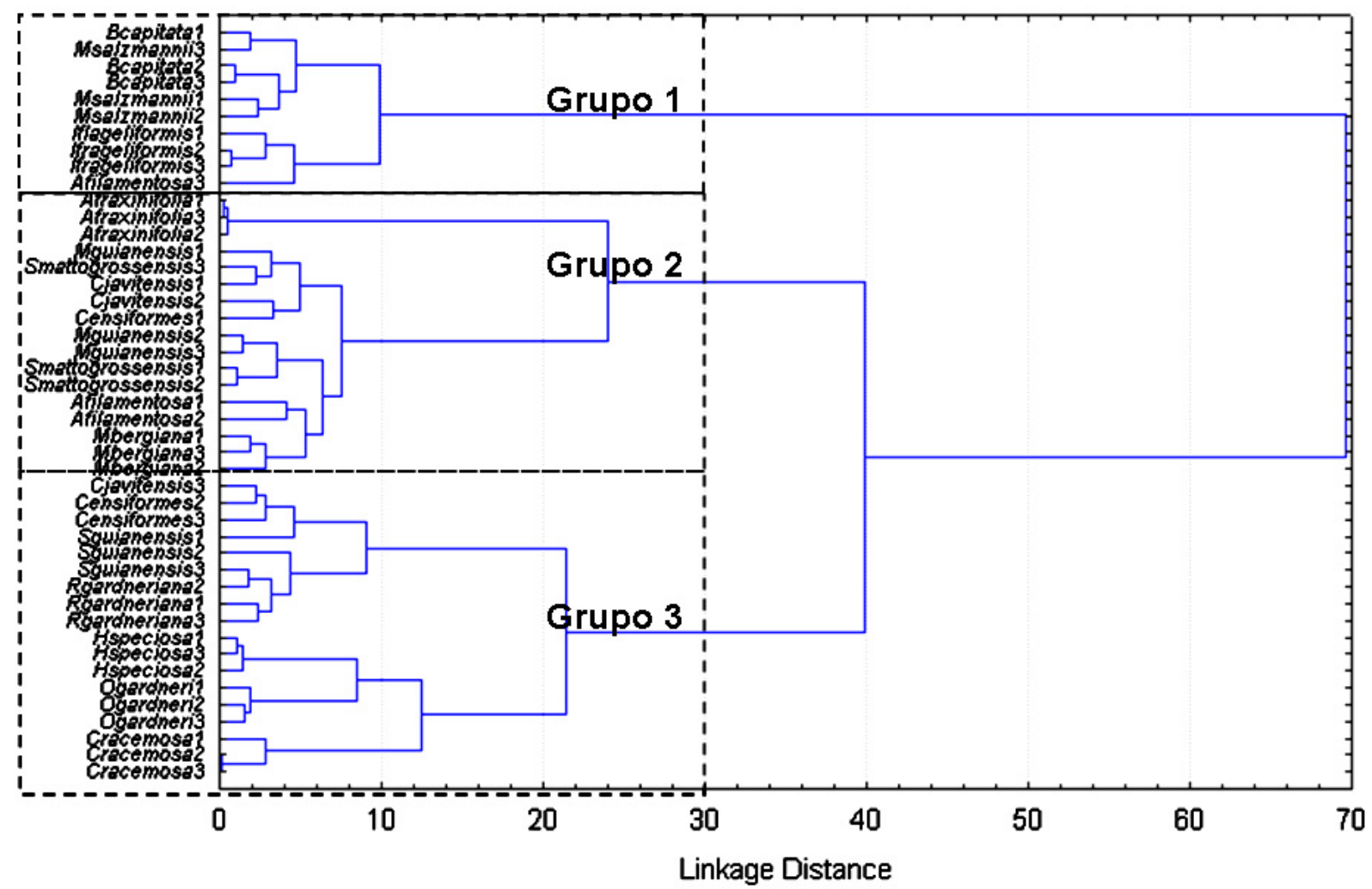

Figura 1. Grupos funcionais vegetais formados a partir de variações nos caracteres morfológicos foliares de espécies dominantes em uma área de restinga em Maracaípe, Pernambuco-Brasil.

O grupo 1 mostrou a menor quantidade de espécies, reunindo todos os indivíduos das espécies Buchenavia capitata, Manilkara salzmannii e Inga flagelliformis, e apenas um único indivíduo da espécie Abarema filamentosa (Tab. 3). O grupo 2 reuniu os indivíduos das espécies Andira fraxinifolia, Myrcia guianensis, Myrcia bergiana e Saccoglotis mattogrossensis, além de dois indivíduos da espécie Abarema filamentosa, dois da espécie Casearia javitensis e apenas um da espécie Chamaecrista ensiformes (Tab. 3). $\mathrm{O}$ agrupamento 3 incluiu os indivíduos das espécies Sloanea guianensis, Rapanea gardneriana, Hancornia speciosa, Ocotea gardneri, Cupania racemosa, um indivíduo da espécie Casearia javitensis e dois da espécie Chamaecrista ensiformes (Tab. 3).

Ao avaliar os grupos formados através da proximidade filogenética observa-se que as espécies analisadas pertencem à classe Magnoliopsida, 
dispostas em quatro de suas subclasses (Cronquist, 1981) (Tab. 3). A maioria das espécies dos grupos um e dois pertencem à subclasse Rosidea, com exceção para a espécie M. salzmannii, no grupo um, e para a espécie C. javitensis, no grupo dois, ambas pertencentes à subclasse Dillenidae. O grupo três apresentou espécies das quatro subclasses encontradas (Tab. 3).

Tabela 3. Classificação taxonômica das espécies de Magnoliopsida pertencentes aos grupos funcionais obtidos através de análise estatística em caracteres morfológicos foliares de espécies dominantes em uma área de restinga em Maracaípe, Pernambuco-Brasil.

\begin{tabular}{|c|c|c|c|c|}
\hline Subclasse & Ordem & Família & Espécie & Indivíduos \\
\hline \multicolumn{5}{|c|}{ Grupo 1} \\
\hline Rosidae & Myrtales & Combretaceae & Buchenavia capitata & $1,2,3$ \\
\hline Rosidae & Rosales & Leg - Mimosaceae & Inga flagelliformis & $1,2,3$ \\
\hline Rosidae & Rosales & Leg - Mimosaceae & Abarema filamentosa & 3 \\
\hline Dillenidae & Ebenales & Sapotaceae & Manilkara salzmannii & $1,2,3$ \\
\hline \multicolumn{5}{|c|}{ Grupo 2} \\
\hline Rosidae & Rosales & Leg - Caesalpinaceae & Chamaecrista ensiformes & 1 \\
\hline Rosidae & Rosales & Leg - Fabaceae & Andira fraxinifolia & $1,2,3$ \\
\hline Dillenidae & Violales & Flacourtiaceae & Casearia javitensis & 1,2 \\
\hline & & & Saccoglotis & $1,2,3$ \\
\hline Rosidae & Linales & Humiriaceae & mattogrossensis & \\
\hline Rosidae & Rosales & Leg - Mimosaceae & Abarema filamentosa & 1,2 \\
\hline Rosidae & Myrtales & Myrtaceae & Myrcia guianensis & $1,2,3$ \\
\hline Rosidae & Myrtales & Myrtaceae & Myrcia bergiana & $1,2,3$ \\
\hline \multicolumn{5}{|c|}{ Grupo 3} \\
\hline Asteridae & Gentianales & Apocynaceae & Hancornia speciosa & $1,2,3$ \\
\hline Rosidae & Rosales & Leg - Caesalpinaceae & Chamaecrista ensiformes & 2,3 \\
\hline Dillenidae & Malvales & Elaeocarpaceae & Sloanea guianensis & $1,2,3$ \\
\hline Dillenidae & Violales & Flacourtiaceae & Casearia javitensis & 3 \\
\hline Magnoliidae & Magnoliales & Lauraceae & Ocotea gardneri & $1,2,3$ \\
\hline Dillenidae & Primulales & Myrsinaceae & Rapanea gardneriana & $1,2,3$ \\
\hline Rosidae & Sapindales & Sapindaceae & Cupania racemosa & $1,2,3$ \\
\hline
\end{tabular}

\section{DISCUSSÃO}

Os caracteres morfológicos foliares apresentam respostas rápidas durante o processo de aclimatação, pois a folha é o órgão que responde mais facilmente às condições do ambiente e apresentam, geneticamente, uma amplitude de tolerância a essas variações que são capazes de promover respostas às mudanças no microambiente, sejam elas grandes ou pequenas. $\mathrm{O}$ conhecimento acerca da amplitude de tolerância das espécies vegetais é restrito, visto que a resposta funcional da espécie é identificada por um grupo de características edafoclimáticas e não apenas por um único 
fator isolado; isto dificulta a identificação do fator ambiental que promove a variação na morfologia foliar.

As espécies analisadas neste estudo estão estabelecidas em uma planície litorânea, sem variações de altitude, o que nos leva a crer que as variações no comprimento do pecíolo, nestes indivíduos, estão relacionadas com a necessidade de uma exposição mais eficiente da lâmina foliar ao sol. Variações na morfometria do pecíolo foram estudadas em florestas de altitude, com o diagnóstico de uma correlação positiva entre a altitude e o comprimento do pecíolo; o comprimento do pecíolo aumentava com a elevação da altitude (Halloy e Mark 1996). Para King e Maindonald (1999), o pecíolo pode se alongar para evitar a sobreposição de folhas em áreas sombreadas da paisagem e aumentar a captação de luz pela lâmina foliar.

Variações na área foliar são descritas na literatura como determinantes nas alterações de taxas de evapotranspiração do ecossistema (Dunn e Connor, 1993; Jayasuriya et al., 1993). Para Niinemets (1999), espécies com menor área foliar tendem a ocupar os espaços na paisagem por crescimento horizontal. Segundo este autor, espécies estabelecidas em florestas úmidas, com uma boa e constante disponibilidade hídrica, apresentam um aumento da área foliar com o aumento da temperatura, promovendo uma maior perda de água por evapotranspiração. Autores como Richardson et al. (2001) informam que um aumento da intensidade luminosa sobre a superfície foliar favorece uma maior expansão da área de sua lâmina, elevando o potencial fotossintético da folha e a perda de água por transpiração.

Espécies estabelecidas em ambientes com reduzida disponibilidade hídrica, como em certos pontos da restinga situados sobre planícies litorâneas no Brasil, com um bom regime de chuvas e solo arenoso que não retém água, mostram uma correlação oposta àquelas observadas com espécies estabelecidas nas florestas úmidas. Em uma paisagem de restinga, as espécies estabelecidas em locais de pleno sol possuem folhas menores do que aquelas que se estabelecem em locais de sombra.

A literatura indica que as análises de agrupamento, baseadas em caracteres vegetativos e na fenologia e história de vida, têm mostrado melhores resultados no agrupamento de espécies com resposta funcional semelhante do que aquelas que consideram os caracteres reprodutivos (Leishman e Westoby, 1992).

$$
\text { Pound e Clements }
$$

reconhecem seis grandes grupos funcionais (plantas lenhosas, semi-arbustos, plantas perenes, ervas, plantas aquáticas e as talófitas). Entretanto, vários outros autores 
têm subdividido esses grupos em outros menores, baseados em altura, hábito de crescimento, história de vida, deciduidade de folhas, entre outros parâmetros (Grisebach, 1872; Warming, 1923; Du Rietz, 1931; Kuchler, 1949; Dansereau, 1951; Ellenberg e Mueller-Dombois, 1967; Box, 1981).

Em regiões semi-áridas, as análises de agrupamento têm demonstrado que, mesmo dentro de um grupo, pequenas variações ainda são observadas (Leishman e Westoby, 1992). Apesar das áreas de restinga não serem classificadas como regiões semi-áridas, existe, nestas áreas, uma deficiência hídrica local, a que pode induzir pequenas respostas individuais, fato que não confere uma homogeneidade maior aos grupos.

As classificações funcionais são utilizadas para descrever variações na vegetação em escala geográfica, principalmente aquelas variações em resposta às condições edafoclimáticas (Leishman e Westoby, 1992). Aparentemente, a localização geográfica dos indivíduos na paisagem condicionou a formação dos grupos. Neste estudo, os indivíduos estabelecidos em locais de borda e de campo aberto na paisagem, com alta luminosidade, formaram o grupo 3, enquanto as espécies dos grupos 1 e 2, apesar de também estarem estabelecidas em ambientes de borda, estas áreas apresentavam uma maior sobreposição de copas, formando regiões mais sombreadas na paisagem.

\section{CONCLUSÃO}

Apesar das espécies dos grupos 1 e 2 serem próximas filogeneticamente, indicando uma resposta genética da espécie as características edafoclimáticas, a heterogeneidade da filogenia observada no grupo 3 indica que este grupo está mais aclimatado as condições do ambiente, independe de sua filogenia.

\section{AGRADECIMENTOS}

Ao Programa de Pós-Graduação em Geografia (PPGEO) da Universidade Federal de Pernambuco (UFPE) e à Fundação de Amparo à Ciência e a Tecnologia do Estado de Pernambuco (FACEPE), pela concessão de bolsa de estudo.

\section{REFERÊNCIAS}

ARAÚJO, D.S.D. \& LACERDA, L.D. A natureza das restingas. Ciência Hoje, 6(33): 42-48. 1987.

ARAÚJO, D.S.D. de. \& HENRIQUES, R.P.B. Análise florística das restingas do estado do Rio de Janeiro. B. In: LACERDA, L.D.; ARAÚJO, D.S.D. de.; CERQUEIRA, R.; Turq, B. (eds.). 
Restingas: Origem, Estrutura e Processos.

Niterói: CEUFF, p.47-60. 1984.

ESTEVES, F.A.; SCARANO, F.R. \& FURTADO, A. Restingas e lagoas costeiras do Norte Fluminense - Site 5. In: Seeliger, U., Cordazzo, C.; Barbosa, F. (eds.). Os Sites e o Programa Brasileiro de Pesquisas Ecológicas de Longa Duração. FURG, UFMG, Belo Horizonte, pp. 83100. 2002.

FURZETO, A. P. \& LOMÔNACO, C. Potencial plástico de Cabralea cajerana e seu papel na formação de ecótipos em área de cerrado e vereda, Uberlândia, MG. Revista Brasileira de Botânica, 23:169176, 2000.

BOEGER, M.R.T. \& WISNIEWSK, C. Comparação da morfologia foliar de espécies arbóreas de três estádios sucessionais distintos de floresta ombrófila densa (Floresta Atlântica) no Sul do Brasil. Revista Brasil. Biol., 26(1): 61-72. 2003.

KLICH, M.G. Leaf variations in Elaeagnus angustifolia related to environmental heterogeneity.

Environmental and Experimental Botany, 44:171-183, 2000.

KONG, H. Z. Comparative morphology of leaf epidermis in the Chloranthaceae.
Botanical Journal of the Linnean Society, 136:279-294. 2001.

MONTEIRO, J.E.B.A.; SENTELHAS, P.C.; CHIAVEGATO, E.J.; GUISELINI, C.; SANTIAGO, A.V. \& PRELA, A. Estimação da área foliar do algodoeiro por meio de dimensões e massa das folhas. Bragantia, 64:15-24. 2005

HENRIQUES, R.P.B.; ARAÚJO, D.S.D. \& HAY, J.D. Descrição e classificação dos tipos de vegetação da restinga de Carapebus, Rio de Janeiro. Revista Brasileira de Botânica, 9:173-189. 1986.

FREIRE, M.S. Levantamento florístico do Parque Estadual das Dunas de Natal. Acta Botanica Brasílica, 4:301-315. 1990.

NIINEMETS, U. Components of leaf dry mass per area - thickness and density alter leaf photosynthetic capacity in reverse directions in wood plants. New Phytologist, 144(1):35-47, 1999.

KING, D. A. \& MAINDONALD, J. H. Tree architecture in relation to leaf dimnsions and tree stature in temperate forest saplings. Journal of Ecology, 87(6): 1012-1024, 1999.

LEISHMAN, M.R. \& WESTOBY, M. Classifying Plants into Groups on the Basis 
RBGF - Revista Brasileira de Geografia Física

Recife-PE Vol.1 n.02 Set/Dez 2008, 50-63

of Associations of Individual

Traits-Evidence from Australian Semi-

Arid Woodlands. The Journal of Ecology,

80(3):417-424. 1992. 